Received: 21 November 2018

Accepted: 12 September 2019

Published online: 02 October 2019

\section{Tradeoffs in hyphal traits determine mycelium architecture in saprobic fungi}

\author{
Anika Lehmann ${ }^{1,2}$, Weishuang Zheng ${ }^{3}$, Katharina Soutschek ${ }^{1}$, Julien Roy ${ }^{1,2}$, Andrey M. Yurkov $\mathbb{D}^{4}$ \\ \& Matthias C. Rillig ${ }^{1,2}$
}

The fungal mycelium represents the essence of the fungal lifestyle, and understanding how a mycelium is constructed is of fundamental importance in fungal biology and ecology. Previous studies have examined initial developmental patterns or focused on a few strains, often mutants of model species, and frequently grown under non-harmonized growth conditions; these factors currently collectively hamper systematic insights into rules of mycelium architecture. To address this, we here use a broader suite of fungi ( 31 species including members of the Ascomycota, Basidiomycota and Mucoromycota), all isolated from the same soil, and tested for ten architectural traits under standardized laboratory conditions. We find great variability in traits among the saprobic fungal species, and detect several clear tradeoffs in mycelial architecture, for example between internodal length and hyphal diameter. Within the constraints so identified, we document otherwise great versatility in mycelium architecture in this set of fungi, and there was no evidence of trait 'syndromes' as might be expected. Our results point to an important dimension of fungal properties with likely consequences for coexistence within local communities, as well as for functional complementarity (e.g. decomposition, soil aggregation).

The mycelium comprises the entirety of the hyphae of a fungus, representing its nutrient-capture and interaction interface, and the infrastructure for transport within the fungal individual. This structure is designed for a dynamic exploratory lifestyle with its ability to reconfigure, fragment and fuse, and represents the very essence of the fungal lifestyle ${ }^{1}$. Understanding basic "rules" of mycelium structure is therefore of fundamental importance for gaining insight into fungal biology and ecology.

The initial development of the mycelium starting from germinating spores has been extensively studied e.g. ${ }^{2,3}$, revealing some general hyphal growth patterns: emerging from a spore, a hypha extends at an exponential rate followed by a constant linear phase until the formation of a new branch is initiated; each new branch itself follows this exponential-linear phase pattern. Additionally, hyphae show negative autotropism and radial orientation away from the colony center ${ }^{3}$, eventually giving rise to the characteristic circular (in 2D) or spherical (in 3D) shape of "colonies" or fungal individuals which can be observed on homogenous growth media (e.g. agar).

Mycologists have also examined the kinetics and branching behavior of fungi and revealed that fungal mycelia undergo changes in growth behavior due to differentiation. In the model organism Neurospora crassa branching angles decrease after approximately $22 \mathrm{~h}$ while hyphal extension rate and diameters increase. Ultimately, the mycelium establishes a hierarchy in which hyphae of higher branching order have decreased hyphal growth rate and diameter in relation to the parental hyphae from which they emerged. As a consequence, the space-filling capacity of the mycelium increases ${ }^{4}$, leading to a maximum surface area while investing in a minimum of hyphal length ${ }^{5}$.

Modelling approaches further contributed to our understanding of how the growth of fungal mycelia is influenced by internal restructuring processes. Internal resource recycling and translocation affects mycelium formation by e.g. increasing hyphal growth rate while decreasing branching rate ${ }^{6,7}$. Additionally, the formation of anastomoses decreases the number of hyphal tips, hence influencing colony growth rate ${ }^{8}$.

\footnotetext{
${ }^{1}$ Freie Universität Berlin, Institut für Biologie, Plant Ecology, Altensteinstr. 6, D-14195, Berlin, Germany. ${ }^{2}$ BerlinBrandenburg Institute of Advanced Biodiversity Research (BBIB), D-14195, Berlin, Germany. ${ }^{3}$ PKU-HKUST ShenZhen-Hong Kong Institution, Shenzhen, 518057, China. ${ }^{4}$ Leibniz Institute DSMZ - German Collection of Microorganisms and Cell Cultures, Inhoffenstraße 7B, D-38124, Braunschweig, Germany. Correspondence and requests for materials should be addressed to A.L. (email: lehmann.anika@googlemail.com)
} 
The majority of studies exploring the rules of mycelium structure draw their conclusions from a limited suite of fungal strains with a typical focus on few fungal model strains (e.g. Rhizoctonia solani and Neurospora crassa) or mutants of the same species, mainly derived from fungal culture collections ${ }^{6-11}$; with only few notable exceptions e.g. ${ }^{12}$. Thus, we are currently lacking a systematic comparison of a range of architectural features, measured under the same, standardized laboratory conditions, on a larger set of fungi from a common ecological context. This is why we currently only have limited knowledge about tradeoffs governing mycelium architecture that could give insight on structural "rules". So far, insights into such tradeoffs come from studying single species or mutants, and this frequently has not resulted in a consensus. For example, the relationship between hyphal diameter and growth rate can be positive (e.g. Bortrytis) or neutral (e.g., Mucor strains) ${ }^{13}$. The same discrepancy holds true for the relationship between hyphal branching frequency (number of hyphal tips) and hyphal growth rate, which was examined in multiple studies focusing on Neurospora strains and mutants: some studies found a positive relationship ${ }^{14}$ and others did not ${ }^{15}$.

Recently, advantages of pursuing a trait-based approach in fungal ecology have been introduced ${ }^{16,17}$. One clear benefit of such an approach is to move beyond idiosyncratic comparisons of a few isolates to make comparisons using larger sets of fungal isolates, offering opportunities for more general inferences about mycelium architectural rules.

Here, we report on studies designed to collect mycelium architecture traits for a set of 31 saprobic fungal strains, containing members of the phyla Ascomycota, Basidiomycota and Mucoromycota which were all isolated from the same soil and hence ecological context.

Our goal was to uncover general "rules" of hyphal growth traits and arrangements within the peripheral zone of an established mycelium by identifying tradeoffs in a large suite of fungi exhibiting a broad spectrum of morphological characteristics.

\section{Materials and Methods}

Fungal strains. Fungal strains were originally cultured from Mallnow Lebus, a dry grassland in a nature conservation reserve (Brandenburg, Germany, $52^{\circ} 27.778^{\prime} \mathrm{N}, 14^{\circ} 29.349^{\prime} \mathrm{E}$ ). A set of 31 fungal strains were isolated from soil samples as described in Andrade-Linares et al. ${ }^{18}$. Briefly, soils were diluted or washed to minimize spore abundance and increase the isolation of fungi derived from hyphae attached to soil particles ${ }^{19,20}$. For isolation a variety of media and antibiotics were used to target Ascomycota, Basidiomycota and Mucoromycota while suppressing bacterial growth. Isolates were incubated at $22^{\circ} \mathrm{C}$ and were cultured on PDA. The fungal set comprised members of the Ascomycota (twenty strains), Basidiomycota (four strains) and Mucoromycota (seven strains) (Fig. 1, Table S1).

Fungal phylogeny. Phylogenetic relationships between the fungal isolates were inferred based on the complete intergenic transcribed spacer (ITS) and a part of the large rRNA subunit (LSU). DNA was extracted using the Qiagen DNeasy PowerSoilKit (100) or MasterPure Yeast DNA Purification Kit (Epicenter, Madison, WI, USA), following the manufacturer's instructions. Amplification of the ITS and partial LSU regions were performed with primers ITS1F and LR5, while internal primers ITS4 and NL4 were used for sequencing with Sanger technology. Sequences were assembled and manually edited to resolve ambiguous base calling using Sequencher 5.4.6 (Gene Codes, Ann Arbor, MI, USA). We used ITS ${ }^{21}$ to split the rRNA sequences into the different subregions ITS1, 5.8S, ITS2 and LSU. Each region was aligned independently using AlignSeqs in the R package "decipher" v.2.0 $0^{22,23}$. Aligned subregion sequences were concatenated. Pairwise distances from sequences were calculated using the JC69 evolutionary model and used to construct a neighbor-joining tree, with the dist.ml and NJ functions respectively, of the R package "phangorn" $2.5 .5^{24}$. Root was placed at the midpoint of the longest path between any two tips. Taxonomic annotations of the fungal isolates were based on each subregion. We used the Naive Bayesian Classifier ${ }^{25}$ as implemented in the R package "dada2"26 against UNITE database for ITS1 or ITS2 ${ }^{27}$, and against the RDP LSU database for $\mathrm{LSU}^{28}$. A confidence threshold was calculated with bootstrap analysis and an annotation was deemed valid when supported in $80 \%$ of the bootstraps. The best resolved taxonomic annotation among the regions was chosen. In the case of a conflict of taxonomic annotations between regions, priority was given to ITS1 or ITS2 because UNITE is more complete than the RDP LSU database. We followed the phylum classification by Spatafora et al. ${ }^{29}$ (Table S1).

Hyphal traits. We conducted two studies to collect traits for the 31 fungal strains. In order to allow meaningful comparisons of collected traits, all studies were performed in the same in vitro system with potato dextrose agar (PDA; Carl Roth $\mathrm{GmbH}$ ) as growth substrate, focusing on traits that were measurable for all strains, and on methods that worked for all. We needed to use a translucent agar growth medium to enable investigation of fungal hyphae; this study would not have been possible in soil with the currently available methods. Furthermore, the application of a single growth medium guaranteed consistent, standardized environmental conditions for the trait measurements ${ }^{16}$. However, results cannot be extended to situations in which other environmental parameters prevail, such as different growth media with distinct sources of nutrition (e.g. C, N, vitamins, amino acids, microelements).

Due to the high variability in colony growth among our 31 fungal strains, the set of traits and locations within the colony that could be measured was naturally restricted. The only reliable inoculation technique that works for this diverse set of fungi uses a colonized poppy seed (as described below), since some of our fungi do not readily sporulate. Since spores are unavailable, we cannot use a single spore as the starting point of mycelial development, as done in studies with selected model strains: we already start with a small mycelium. This, in turn, necessitated a focus on the colony edge since in most of our fungal strains the mycelium behind this peripheral zone was too dense to be resolved for identification of single hyphae (even in the relative small observation system we used, see below). Hence, it was not possible to investigate hyphal branching hierarchies. Additionally, some basic hyphal 


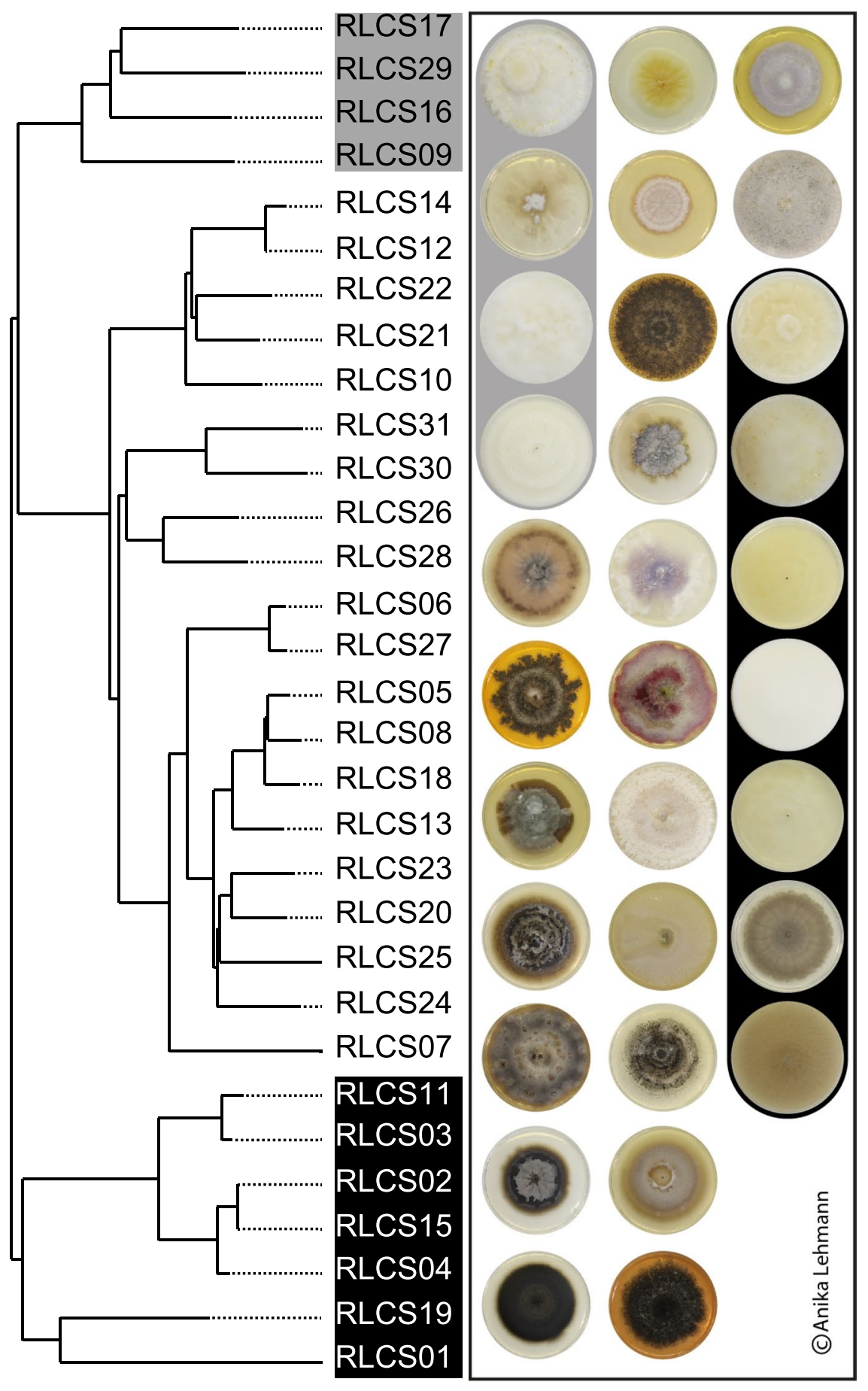

Figure 1. Phylogenetic tree of the 31 fungal strains belonging to the phyla Ascomycota, Basidiomycota and Mucoromycota. Colony pictures are from four-week old cultures grown on potato dextrose agar, and their order follows the order of the phylogenetic tree. Further information about phylogeny and accession numbers of the 31 strains are available in Table S1. Information about phylum affiliation is color-coded (black: Mucoromycota, grey: Basidiomycota, white: Ascomycota).

traits (e.g. hyphal tip growth) were only measurable for a subset of strains (preliminary experiment; data not shown) and hence were not further considered.

We found ten traits to be measurable for all 31 fungal strains in the established in vitro system. We focused on hyphal growth traits and traits representing hyphal arrangements within the mycelium by using fractal dimension. We believe these traits are also informative for key ecosystem functions related to mycelium architecture, such as soil aggregation ${ }^{17}$. For the hyphal growth traits, we focused on measuring hyphal branching angle (BA), internodal 
length (IL) and diameter (D). For this, in the first study, the fungal strains were grown on single concavity slides carrying $150 \mu \mathrm{l}$ of PDA; a widely used standard growth medium for filamentous, saprobic fungi. We chose to reduce the concentration of PDA to $10 \%$ to obtain nutrient reduced growth medium for reduced mycelial density. This was necessary to be able to identify single hyphae in very densely growing fungi. To guarantee solidification of the medium, we added agar (Panreac AppliChem) to reach $15 \mathrm{gL}^{-1}$ concentration. The growth medium was flattened by placing a cover slip on the liquid medium drop until it solidified. A pre-sterilized poppy seed carrying the target fungal strain was positioned in the center of the concavity. The slide was placed in a $9 \mathrm{~cm}$ Petri dish filled with a $5 \mathrm{~mm}$ layer of water agar to maintain high air humidity. Plates were sealed and stored at room temperature $\left(22^{\circ} \mathrm{C}\right)$ in the dark until the fungal colony covered half of the concavity area. For each fungal strain $(n=31)$, five slides were prepared and placed in separate Petri dishes. Hence, we had five replicates for 31 fungal strains yielding 155 concavity slides for analyses. For the measurements, slides were examined under the microscope (Leica DM2500, bright field, 200x). Per slide, we randomly chose five hyphae as subsamples; for each of these hyphae we measured at the colony edge the last developed branching angle, the internodal lengths between this last and second-to-last branch and the hyphal diameters within this youngest internodal segment. For analyses, we used the image processing software Image $\mathrm{J}^{30}$. For each replicate for each fungal strains, we calculated a mean value and a coefficient of variation $(\mathrm{CV})$ from the subsample data. These represented two aspects of a trait: the average value and its variability. The trait data used in statistical analyses were the average of mean values and CVs of the five replicates (i.e. $n=31$ ).

In the second study, we investigated the complexity and the heterogeneity of fungal mycelia by applying fractal analysis - a technique used to assess self-similarity and space-filling capacity of fungal hypha ${ }^{31}$ to cover the hyphal arrangement. For this, we applied the same approach as in the first experiment but with eight replicates per fungal strain yielding 248 concavity slides for analyses. At harvest, the slides were examined under the microscope (Leica DM2500, bright field, 200x) focusing on the outer $200 \mu \mathrm{m}$ of the growing zone to investigate the "surface fractals" 32 . Camera (Leica DFC290) settings were chosen to generate grayscale photos with high contrast. For each slide, we photographed three randomly chosen fields of view at the colony edge. These settings and further image processing in Image $\mathrm{J}^{30}$ and Adobe Illustrator (CS6, v.16.0.0) were necessary to guarantee comparable and unbiased photos that can be processed by image analysis software. First, photos were converted to 8-bit binary images in ImageJ and subsequently hyphae were skeletonized. For this, a thinning algorithm repeatedly reduced pixels from the edge of the target object until a one-pixel wide shape was reached ${ }^{33}$. In Illustrator, the skeletonized hyphae were reconnected and image artifacts excluded, if necessary. Line thickness was adjusted to mean diameter trait values derived from experiment one. The final processed images were loaded into the ImageJ plug-in "FracLac" 34 to measure fractal dimensions. We chose box counting dimensions (Db) as a measure of structural complexity (i.e. the degree of detail or amount of parts a pattern consists of), and lacunarity (L) as a representative of structural heterogeneity (i.e. the gappiness or "rotational and translational invariance" in a pattern ${ }^{34}$ ). In FracLac, we used overall default settings but for the grid design we allowed for multiple rotational orientations in the analyses. Finally, subsample data were used to calculate CVs for box counting dimension and lacunarity. The subsample data were then merged to one mean and CV trait value per replicate. Additionally, we verified if implementing diameter data altered fractal dimension data by correlating skeletonized and adjusted diameter data for both box counting dimension and lacunarity (Fig. S1).

Statistics. We analyzed the relationships between the ten architectural trait variables derived from 31 saprobic fungal strains represented by both mean value and coefficients of variation $(C V)(n=31)$. First, to evaluate fungal distribution in ten-dimensional trait space, we ran a principal component analysis using the function $\operatorname{prcomp}()$ in the package "stats" with z-transformed data. Significance of PC axes was determined via the function testdim ()$^{35}$ in the package "ade4"36-38. Only the first axis was significant, hence we included PC axis 1 and 2 in the visualizations without losing information from the excluded axes. Next, we conducted kernel density estimation to assess species occurrence probability following the procedure presented by Diaz et al..$^{39}$. Briefly, we used two most important PC axes (axis 1 and 2) to run a two-dimensional kernel estimation by applying the kde() function in the "ks" package. To do so, the optimal kernel smoother had to be chosen by using the Hpi() function in the package "ks" on the two PC axes ${ }^{40}$; following Diaz et al..$^{39}$, we chose unconstrained bandwidth selectors. We constructed the contour plots from the estimated kernel density distribution. The depicted contour lines (by contourLevels() function) and corresponding color gradient represent the 0.5 and 0.95 quantiles of the probability distribution.

Second, to test for phylogenetic signal in our trait variables we used Moran's I statistic, a measure for phylogenetic autocorrelation, as implemented in the package "phylosignal". We accounted for phylogenetic relatedness among species (indicated by detected phylogenetic signals) by calculating phylogenetically independent contrast of our trait variables with the packages "picante" 41 and "ape" 42 using the functions pic() and match.phylo.data(). We evaluated if the assumptions of the Brownian motion model were satisfied by our data ${ }^{43}$. For that, we investigated the standardization of the contrasts via diagnostic regression tests to evaluate the relationship between absolute standardized contrasts and (i) the square root of their standard deviation ${ }^{44}$ and (ii) the node height (i.e. node age ${ }^{45,46}$ ). Identified influential nodes (node 37 (strains RLCS02 and RLCS15) and node 57 (strains RLCS21 and RLCS22 $)^{47}$ ) were excluded, following the threshold of absolute studentized residuals greater than $3^{44,48}$. To satisfy the Brownian motion model assumption, we further used log transformed trait values.

Third, multiple pairwise correlations using Pearson's rho were conducted and plotted with the function corr$\operatorname{plot}()$ in the eponymous package ${ }^{49}$. Analyses were done for original (non-transformed, $\mathrm{n}=31$ ) and phylogenetically corrected data (log-transformed, $\mathrm{n}=28$ ).

Fourth, we ran linear regressions and further investigated the relationships by quantile regression with the package "quantreg" (https://github.com/cran/quantreg). Under most ecological conditions, linear regressions tend to over- or underestimate relationships due to a focus on the mean of the response distribution. Especially in wedge-shaped data distributions, indicating unmeasured limiting factors, quantile regressions are more informative since they test the relationship between response and predictor variable at their maxima ${ }^{50,51}$. Both regression 

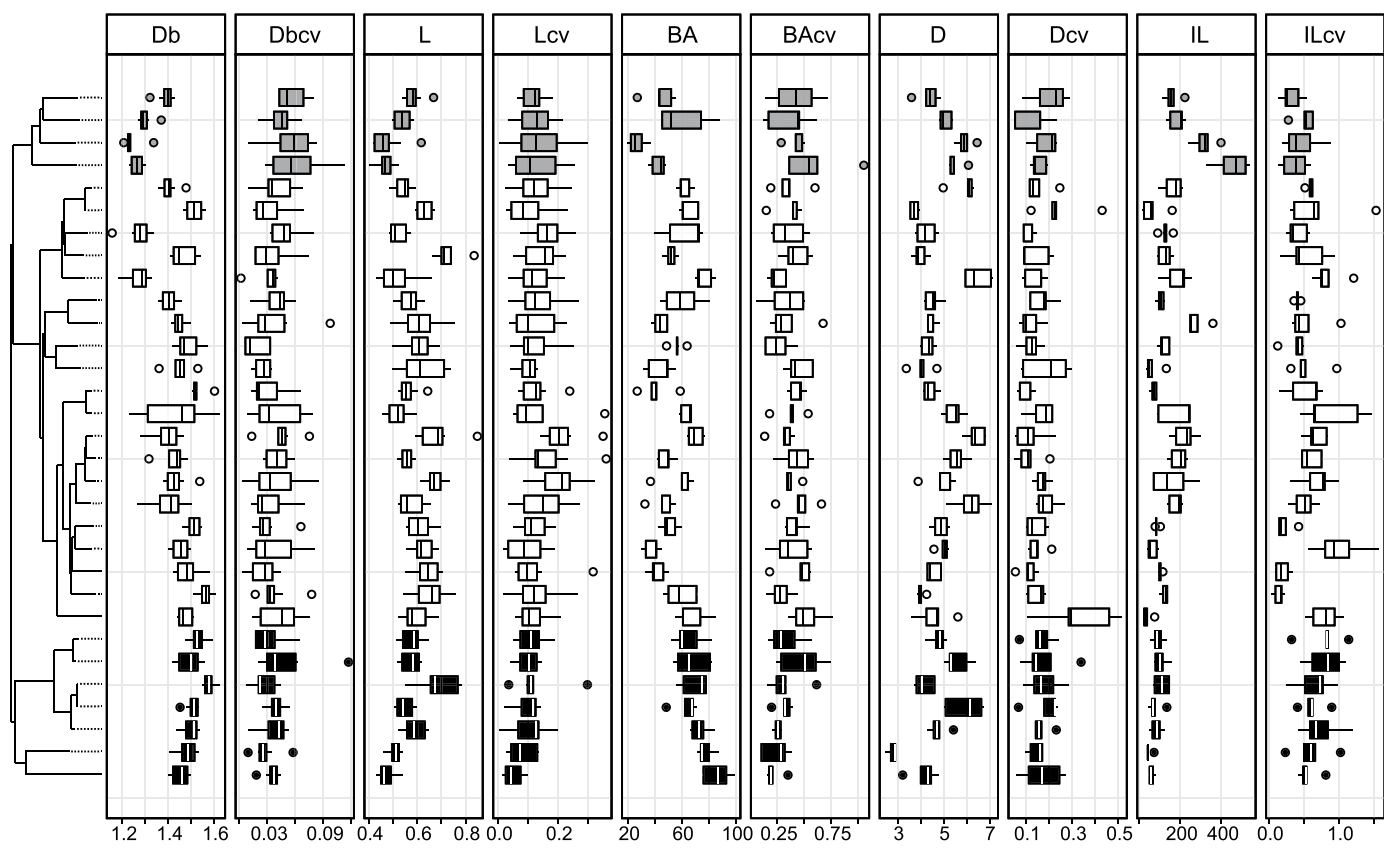

Figure 2. Distributions of our ten trait variables, mean value and their coefficient of variation (CV) measured in this study: box counting dimension (unitless, $\mathrm{Db}$ with $\mathrm{n}=8, \mathrm{Db}_{\mathrm{CV}}$ with $\mathrm{n}=8$ ), lacunarity (unitless, $\mathrm{L}$ with $\mathrm{n}=8, \mathrm{~L}_{\mathrm{CV}}$ with $\mathrm{n}=8$ ), branching angle (in ${ }^{\circ}, \mathrm{BA}$ with $\mathrm{n}=5, \mathrm{BA}_{\mathrm{CV}}$ with $\mathrm{n}=5$ ), hyphal diameter (in $\mu \mathrm{m}, \mathrm{D}$ with $\mathrm{n}=5, \mathrm{D}_{\mathrm{CV}}$ with $\mathrm{n}=5$ ), internodal length (in $\mu \mathrm{m}$, IL with $\mathrm{n}=5, \mathrm{IL}_{\mathrm{CV}}$ with $\mathrm{n}=5$ ). The Tukey boxplots represent 25th and 75th percentile, median and outlying points. Information about phylum affiliation is color-coded (black: Mucoromycota, grey: Basidiomycota, white: Ascomycota).

analyses were run on z-transformed data and model residuals were tested for homogeneity and normal distribution. Additionally, we ran multiple pairwise regressions for both original and phylogenetically corrected data to provide graphical information on data distributions of all trait combinations (see Supplementary Material). These were generated by the function ggpairs() of the package GGally ${ }^{52}$.

All analyses were conducted in R (v. 3.4.1 $1^{53}$ ) and plots were created with the graphics package ggplot $2^{54}$ and its extension GGally.

\section{Results and Discussion}

Trait expression. Overall, we found variability among strains for all traits (Fig. 2). The application of fractal dimensions on mycelium structure revealed that trait mean values of box counting dimensions (Db) ranged between 1.2 and 1.6, where a value of 1 represents a single unbranched hypha, and a value of 2 a complex space-filling mycelium. The most complex mycelium was found in the Mucoromycota, while Basidiomycota had the most simply structured mycelia (see also Table S2 and Fig. S2). For lacunarity (L), we found in our study that trait values ranged between 0.4 (Basidiomycota) and 0.7 (Ascomycota). With increasing trait value, the heterogeneity and hence gappiness of the mycelium increased. The investigation of hyphal features revealed that the branching angle (BA) varied substantially across fungal strains from 26 to $86^{\circ}$ with Mucoromycota having large angles and Basidiomycota rather small angles. For hyphal diameter (D) trait values ranged from 2.7 to $6.5 \mu \mathrm{m}$ across the 31 strains where both extremes could be found in the Mucoromycota. The length of the hyphal internodes (IL) showed considerable differences: Within Basidiomycota internodal lengths of $453 \mu \mathrm{m}$ could be reached while in Mucoromycota the lowest value of $40 \mu \mathrm{m}$ was measured. Our values are within the range of previously reported architectural features of selected, individual saprobic filamentous fungi e.g. ${ }^{32,55-59}$. It is important to note that the interpretation of our trait data is limited to these 31 fungal strains under defined environmental conditions. For future work, it would be interesting to examine if fungal lifestyle (e.g. plant pathogens, decomposer, or primarily soil-inhabiting fungi) influences the expression of the traits we examined here.

After establishing the trait database, we investigated the trait space generated by the collected fungal architectural features. To do this, we applied principal component analyses.

PCA. For our 31 fungal strains, the sole significant first principal components accounted for $34 \%$ of the variability in the ten architecture traits (Fig. 3A). In this ten-dimensional trait space, the set of our 31 fungal strains occupied the whole PC plane with a clear separation of the three phyla across the plane. Considering the sole significant PC axis 1 (Table S3), Ascomycota assembled in the center flanked by Mucoromycota on the left, driven by large branching angles and high mycelial complexity, and Basidiomycota on the right portion, primarily characterized by long internodes and wide hyphal diameters (Figs 3A and S3). Across species, some clear correlations among traits became visible; hence, we further investigated the type and intensity of potential architectural tradeoffs for our fungal set. 

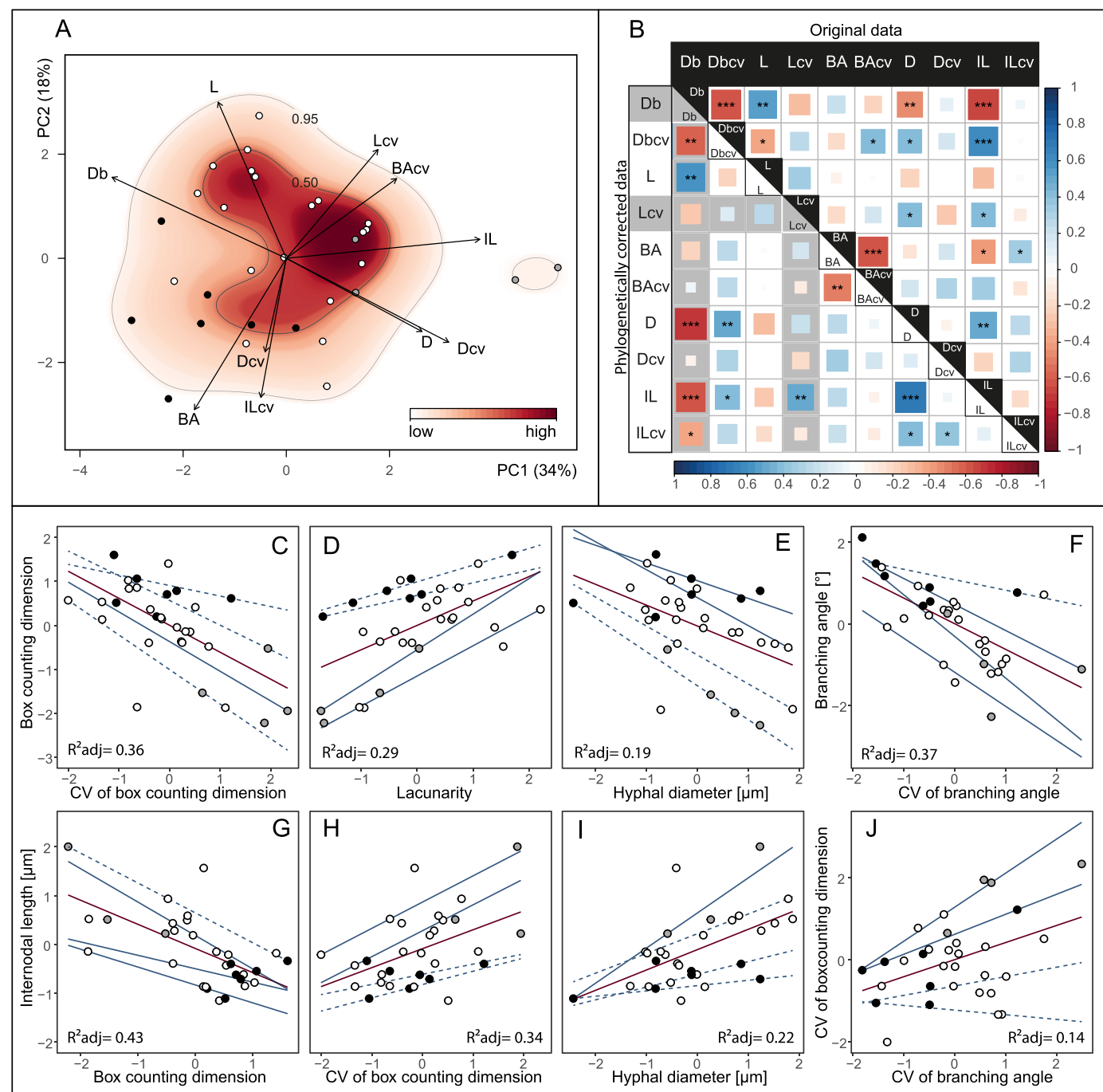

Legend

- Ascomycota

- Basidiomycota

- Mucoromycotina

Figure 3. Outcomes of principal component analysis, trait correlation, linear and quantile regression of the investigated architectural traits. Analyses were conducted on trait data $(n=31)$. (A) Projection of the ordinated 31 fungal strains onto ten architectural trait variables (mean and CV): box counting dimension (Db), lacunarity (L), branching angle (BA), hyphal diameter (D), internodal length (IL) into two-dimensional trait space represented by principal component axis 1 and 2 (explaining 34 and $18 \%$ of variance, respectively). Arrows indicate direction and weight of trait vectors. Color gradient represents probability of species occurrence (white $=$ low, red $=$ high) in the trait space, with the contour lines denoting the 0.50 and 0.95 quantiles of kernel density estimation (see materials and methods section). Information about phylum affiliation is color-coded (black: Mucoromycota, grey: Basidiomycota, white: Ascomycota). (B) Correlation plot of five architectural trait variables and their coefficients of variation. The upper triangle displays original while the lower triangle represents phylogenetically corrected data correlations. Color gradient and square size are proportional to the correlation coefficient (Pearson's rho). Asterisks denote significance level: $* * *<0.001, * *<0.01, *<0.05$. In grey, we highlight trait combinations affected by detected phylogenetic signal (Table S4). (C-J) The eight strongest trait relationships for either original and/or phylogenetically corrected data. Red lines represent linear regression lines and blue lines quantile regression lines, while line type depicts significance of regression lines; solid lines $p$-value $<0.05$, dashed lines $>0.05$. Corresponding regression statistics can be found in Table S5. Adjusted $\mathrm{R}^{2}$ values correspond to linear regressions. Trait data were $\mathrm{z}$-transformed (see Materials and Methods section).

Phylogenetic signal. For this, we first tested all ten traits for a phylogenetic signal to evaluate if the phylogenetic relatedness among fungal strains can influence any trait relationships we want to investigate. Applying Moran's I statistics (Table S4), we found phylogenetic signals in $\mathrm{Db}(\mathrm{I}=0.09, \mathrm{p}=0.04)$ and $\mathrm{L}_{\mathrm{CV}}(\mathrm{I}=0.10, \mathrm{p}=0.04)$. Hence, we needed to account for phylogenetic relations for these two traits among our 31 fungal strains by applying phylogenetically independent contrast in the following analyses. 
Tradeoffs. We found 14 trait pairs with significant correlations of which ten passed phylogenetic correction (Figs 3b, S4 and S5). The strongest correlations were detected between mycelium complexity and its coefficient of variation $\left(\mathrm{Db}-\mathrm{Db}_{\mathrm{CV}}\right.$ in Fig. $\left.3 \mathrm{C}\right)$, mycelium heterogeneity, as measured by lacunarity, (Db - L in Fig. 3D) and hyphal diameter ( $\mathrm{Db}-\mathrm{D}$ in Fig. $3 \mathrm{E})$, as well as between branching angle and its coefficient of variation $\left(\mathrm{BA}-\mathrm{BA}_{\mathrm{CV}}\right.$ in Fig. 3F). For internodal length, we detected relationships with mycelium complexity (IL - Db in Fig. 3G), variability in mycelium complexity (IL - $\mathrm{Db}_{\mathrm{CV}}$ in Fig. $3 \mathrm{H}$ ) and hyphal diameter (IL - D in Fig. 3I). Another strong correlation was found between the coefficients of variation of mycelium complexity and branching angle $\left(\mathrm{Db}_{\mathrm{CV}}\right.$ - $\mathrm{BA}_{\mathrm{CV}}$ in Fig. 3J). In addition, weak correlations were found for hyphal diameter and the coefficients of variation of mycelium complexity $\left(\mathrm{D}-\mathrm{Db}_{\mathrm{CV}}\right)$, hyphal diameter and the coefficients of variation of lacunarity $\left(\mathrm{D}-\mathrm{L}_{\mathrm{CV}}\right)$, as well as between internodal length and the coefficients of variation of lacunarity (IL - $\mathrm{L}_{\mathrm{CV}}$ ), internodal length and branching anlge (IL - BA) and the coefficients of variation of mycelium complexity (L - DbCV) and also branching angle and the coefficients of variation of intermodal length (BA - ILCV) (Figs S4 and S5). From these correlations we can deduce multiple rules for mycelium architecture.

For structural complexity (as represented by box counting dimensions) and branching angle, we detected a negative relationship between their mean values and $\mathrm{CVs}\left(\mathrm{Db}-\mathrm{Db}_{\mathrm{CV}}\right.$ in Fig. $3 \mathrm{C}$ and $\mathrm{BA}-\mathrm{BA}_{\mathrm{CV}}$ in Fig. $\left.3 \mathrm{~F}\right)$. Thus strains exhibiting a high trait value for branching angle or mycelium complexity are restricted to this high value, while strains with low values in these traits are capable of further adjusting these features.

Within strains, variability in mycelial complexity itself is determined by increasing internodal length (IL $\mathrm{Db}_{\mathrm{CV}}$ in Fig. $\left.3 \mathrm{H}\right)$ and higher flexibility in branching angle measures $\left(\mathrm{Db}_{\mathrm{CV}}-\mathrm{BA}_{\mathrm{CV}}\right.$ in Fig. 3J). Thus, the degree of mycelial complexity can be modulated via branching patterns (e.g. distance between branches).

Considering the complexity - the space-filling capacity - of a mycelium, we found that more complex mycelia are more heterogeneously structured (Db - L in Fig. 3D). Mycelia with high space-filling capacity tend to be rather heterogeneous in their structure, i.e. their mycelium is not uniformly complex but rather exhibits complex zones replaced by more simple mycelium structures towards the growing edge. At the colony edge, hyphae are confronted with new resources and environmental conditions for which a maximum of flexibility is likely advantageous. Furthermore, complex mycelia have smaller hyphal diameters (Db - D in Fig. 3E) and shorter internodal length (IL - Db in Fig. 3G). A mycelium with long internodes is characterized by less branching and hence less space-filling. However, to be capable of growing long internodes the mycelium needs to improve its structural support, i.e. its tear-resistance. Long hyphae are at risk of fragmentation by shear-stresses ${ }^{60}$. To deal with this risk, hyphal cell walls can thicken and/or hyphal diameter can increase ${ }^{10,61}$. This is congruent with our finding that long internodes are linked with larger hyphal diameters (IL - D in Fig. 3I).

For the other detected traits relationships (D - Dbcv, D - Lcv, IL- Lcv, IL - BA, L - Dbcv, BA - ILcv), no clear ecological or evolutionary explanation is available. It is noteworthy that these traits were of low statistical significance. It is thus necessary to challenge these trait relationships in future works to unravel their true significance.

It is interesting that there were no sharp boundaries in the sense of architectural "syndromes" or clear groups of traits, but rather relatively gradual changes in trait values within the set of fungal isolates we examined. This illustrates the relative versatility of the mycelial growth form in evolutionary terms, at least in the peripheral growth zone of the fungus under experimental conditions, which we examined here. We clearly show that there are limits to how a mycelium can be constructed, since some trait combinations are evidently not favorable (e.g. long internodes and small diameters). However, fungi have evidently otherwise filled the trait space within the constraints of such fundamental tradeoffs, even seen in a sample of 31 species. It will be interesting and necessary, especially for broader external validity beyond the environment from which these strains came, to compare our results to other sets of fungi once such data are available: our results suggest key parameters on which to focus. Ultimately, new knowledge will be gained by the synthesis of the combined research efforts which may yield insight into a hyphal/mycelium architecture spectrum.

\section{Conclusion}

One of the most fundamental decisions a growing hypha has to make is when to branch. Thus, it is maybe not surprising that internodal length was a highly influential variable (aligned with PC axis 1, Fig. 3) in understanding the architecture of mycelia in trait space. This suggests that the trait internodal length is a main driver of mycelium architecture. Mycelia with short internodes can branch more frequently thus developing a more complex mycelium than those with long internodes. However, the capability of growing long unbranched hyphae enables the mycelium to more flexibly adjust their mycelial modules (see positive correlations between IL and $\mathrm{Db}_{\mathrm{CV}}, \mathrm{L}_{\mathrm{CV}}$ ) in response to environmental conditions.

Mycelial architecture is a fundamental property of filamentous fungi, governing the way these organisms explore their substrate. Using a set of fungi co-occurring in the same soil, we show that architectural features vary strongly and reproducibly among different isolates under the same laboratory conditions. It is therefore highly likely that such differences contribute to enabling coexistence within fungal communities ${ }^{62}$ by offering fungi different ways to forage and colonize the soil environment. On the other hand, such trait divergence can also mediate functional complementarity, for example in efficient decomposition of organic material or soil aggregation ${ }^{17}$.

\section{Data Availability}

All data generated or analyzed during this study are included within the paper and its Supplementary Data Files.

\section{References}

1. Wessels, J. G. H. Fungi in their own right. Fungal Genet. Biol. 27, 134-145 (1999).

2. Plomley, N. J. B. Formation of the colony in the fungus Chaetomium. Aust J Biol Sci 12, 53-64 (1959).

3. Trinci, A. P. J. A study of the kinetics of hyphal extension and branch initiation of fungal mycelia. J Gent Microbiol 81, 225-236 (1974). 
4. Barry, D. J. Quantifying the branching frequency of virtual filamentous microbes using fractal analysis. Biotechnol. Bioeng. 110, 437-447 (2013).

5. Moore, D., Robson, G. D. \& Trinci, A. P. J. 21st century guidebook to fungi. 1 edn, (Cambridge University Press, 2011).

6. Boswell, G. P., Jacobs, H., Ritz, K., Gadd, G. M. \& Davidson, F. A. The development of fungal networks in complex environments. Bull. Math. Biol. 69, 605-634 (2007).

7. Falconer, R. E., Bown, J. L., White, N. A. \& Crawford, J. W. Biomass recycling and the origin of phenotype in fungal mycelia. Proc Biol Sci 272, 1727-1734 (2005).

8. Boswell, G. P., Jacobs, H., Davidson, F. A., Gadd, G. M. \& Ritz, K. Functional consequences of nutrient translocation in mycelial fungi. J. Theor. Biol. 217, 459-477 (2002).

9. Butler, G. M. Growth of hyphal branching systems in Coprinus disseminatus. Ann. Bot. 25, 341-\& (1961).

10. McLean, K. M. \& Prosser, J. I. Development of vegetative mycelium during colony growth of Neurospora crassa. T Brit Mycol Soc 88, 489-495 (1987)

11. Robinson, P. M. \& Smith, J. M. Apical branch formation and cyclic development in Geotrichum candidum. T Brit Mycol Soc 75, 233-238 (1980)

12. Vidal-Diez de Ulzurrun, G. et al. Automated image-based analysis of spatio-temporal fungal dynamics. Fungal Genet. Biol. 84, $12-25(2015)$

13. Carlile, M. J., Watkinson, S. C. \& Gooday, G. W. The fungi. 2nd edn, (Academic Press, 2001).

14. Katz, D., Goldstein, D. \& Rosenberger, R. F. Model for branch initiation in Aspergillus nidulans based on measurements of growth parameters. J. Bacteriol. 109, 1097-1100 (1972).

15. Watters, M. K., Lindamood, E., Meunich, M. \& Vetor, R. Strain-dependent eelationship between growth rate and hyphal branching in Neurospora crassa. P Indiana Acad Sci 117, 1-6 (2008).

16. Aguilar-Trigueros, C. A. et al. Branching out: Towards a trait-based understanding of fungal ecology. Fungal Biol Rev 29, 34-41 (2015).

17. Lehmann, A. \& Rillig, M. C. Understanding mechanisms of soil biota involvement in soil aggregation: A way forward with saprobic fungi? Soil Biol. Biochem. 88, 298-302 (2015).

18. Andrade-Linares, D. R., Veresoglou, S. D. \& Rillig, M. C. Temperature priming and memory in soil filamentous fungi. Fungal Ecol 21, 10-15 (2016).

19. Gams, W. \& Domsch, K. H. Beitrage zur Anwendung der Bodenwaschtechnik für die Isolierung von Bodenpilzen. Arch. Mikrobiol. 58, 134-144 (1967).

20. Thorn, R. G., Reddy, C. A., Harris, D. \& Paul, E. A. Isolation of saprophytic basidiomycetes from soil. Appl. Environ. Microbiol. 62, 4288-4292 (1996).

21. Bengtsson-Palme, J. et al. Improved software detection and extraction of ITS1 and ITS2 from ribosomal ITS sequences of fungi and other eukaryotes for analysis of environmental sequencing data. Methods Ecol Evol 4, 914-919 (2013).

22. Wright, E. S. DECIPHER: harnessing local sequence context to improve protein multiple sequence alignment. BMC Bioinformatics 16, 322 (2015).

23. Wright, E. S. Using DECIPHER v2.0 to analyze big biological sequence data in R. R Journal 8, 352-359 (2016).

24. Schliep, K. P. phangorn: phylogenetic analysis in R. Bioinformatics 27, 592-593 (2011).

25. Wang, Q., Garrity, G. M., Tiedje, J. M. \& Cole, J. R. Naive Bayesian classifier for rapid assignment of rRNA sequences into the new bacterial taxonomy. Appl. Environ. Microbiol. 73, 5261-5267 (2007)

26. Callahan, B. J. et al. DADA2: High-resolution sample inference from Illumina amplicon data. Nat. Methods 13, 581-583 (2016)

27. Nilsson, R. H. et al. The UNITE database for molecular identification of fungi: handling dark taxa and parallel taxonomic classifications. Nucleic Acids Res. 47, D259-D264 (2019).

28. Cole, J. R., Wang, Q., Chai, B. \& Tiedje, J. M. In Handbook of Molecular Microbial Ecology I 313-324 (2011)

29. Spatafora, J. W. et al. The fungal tree of life: From molecular systematics to genome-scale phylogenies. Microbiology Spectrum 5 (2017).

30. Schneider, C. A., Rasband, W. S. \& Eliceiri, K. W. NIH Image to ImageJ: 25 years of image analysis. Nat. Methods 9, 671-675 (2012).

31. Posser, J. I. Kinetics of filamentous growth and branching. Gow, N. A. R. \& Gadd, G. M. The growing fungus. 1 edn (Springer, 1995).

32. Obert, M., Pfeifer, P. \& Sernetz, M. Microbial growth patterns described by fractal geometry. J. Bacteriol. 172, 1180-1185 (1990).

33. Zhang, T. Y. \& Suen, C. Y. A fast parallel algorithm for thinning digital patterns. Communications of the Acm 27, 236-239 (1984).

34. Karperien, A. FracLac for ImageJ (1999-2013).

35. Dray, S. On the number of principal components: A test of dimensionality based on measurements of similarity between matrices. Comput. Stat. Data Anal. 52, 2228-2237 (2008).

36. Chessel, D., Dufour, A. B. \& Thioulouse, J. The ade4 package - I: One-table methods. R News 4, 5-10 (2004).

37. Dray, S. \& Dufour, A. B. The ade4 package: Implementing the duality diagram for ecologists. J Stat Softw 22, 1-20 (2007).

38. Dray, S., Dufour, A. B. \& Chessel, D. The ade4 package-II: Two-table and K-table methods. R News 7, 47-52 (2007).

39. Diaz, S. et al. The global spectrum of plant form and function. Nature 529, 167-173 (2016).

40. Duong, T. ks: Kernel smoothing v. R package version 1.11.0 (2018).

41. Kembel, S. W. et al. Picante: R tools for integrating phylogenies and ecology. Bioinformatics 26, 1463-1464 (2010).

42. Paradis, E., Claude, J. \& Strimmer, K. APE: Analyses of Phylogenetics and Evolution in R language. Bioinformatics 20, 289-290 (2004).

43. Cooper, N., Thomas, G. H. \& FitzJohn, R. G. Shedding light on the "dark side' of phylogenetic comparative methods. Methods Ecol Evol 7, 693-699 (2016).

44. Garland, T., Harvey, P. H. \& Ives, A. R. Procedures for the analysis of comparative data using phylogenetically indpendent contrasts. Syst. Biol. 41, 18-32 (1992).

45. Freckleton, R. P. \& Harvey, P. H. Detecting non-Brownian trait evolution in adaptive radiations. PLoS Biol. 4, 2104-2111 (2006).

46. Grafen, A. The phylogenetic regression. Philos T Roy Soc B 326, 119-157 (1989).

47. Orme, D. The caper package: comparative analysis of phylogenetics and evolution in R v. 1.0.1 (2013).

48. Jones, K. E. \& Purvis, A. An optimum body size for mammals? Comparative evidence from bats. Funct. Ecol. 11, 751-756 (1997).

49. Wei, T. \& Simko, V. R package "corrplot": Visualization of a Correlation Matrix v. 0.84 (2017).

50. Cade, B. S. \& Noon, B. R. A gentle introduction to quantile regression for ecologists. Front. Ecol. Environ. 1, 412-420 (2003).

51. Cade, B. S., Terrell, J. W. \& Schroeder, R. L. Estimating effects of limiting factors with regression quantiles. Ecology 80, 311-323 (1999).

52. Schloerke, B. et al. GGally: Extension to 'ggplot2' v. 1.3.2 (2017).

53. R Development Core Team. R: A language and environment for statistical computing v. 3.4.1 (2014).

54. Wickham, H. ggplot2: Elegant graphics for data analysis. (Springer, 2009).

55. Henis, Y., Okon, Y. \& Chet, I. Relationship between early hyphal branching and formation of sclerotia in Sclerotium rolfsii. J Gen Microbiol 79, 147-150 (1973).

56. Ho, H. H. Hyphal branching systems in Phytophthora and other Phcomycetes. Mycopathologia 64, 83-86 (1978).

57. Hutchinson, S. A., Sharma, P., Clarke, K. R. \& Macdonald, I. Control of hyphal orientation in colonies of Mucor hiemalis. T Brit Mycol Soc 75, 177-191 (1980). 
58. Kotov, V., Anishchenko, I., Sirenko, I. \& Reshetnikov, S. Statistical analysis of structural and kinetic characteristics of fungal colony growth with Trichoderma viride Pers.: S.F. Gray. Microbiol. Res. 160, 273-278 (2005).

59. Trinci, A. P. J. A kinetic study of the growth of Aspergillus nidulans and other fungi. J Gent Microbiol 57, 11-24 (1969).

60. Suijdam, J. C. \& van Metz, B. Fungal pellet breakup as a function of shear in a fermentor. J Ferment Technol 59, 329-333 (1981).

61. Trinci, A. P. J. \& Collinge, A. J. Hyphal wall growth in Neurospora crassa and Geotrichum candidum. J Gen Microbiol 91, 355-361 (1975).

62. Crowther, T. W. et al. Untangling the fungal niche: the trait-based approach. Front Microbiol 5, 1-12 (2014).

\section{Acknowledgements}

This work was supported by the Deutsche Forschungsgemeinschaft (RI 1815/16-1). MCR additionally acknowledges an ERC Advanced Grant (694368).

\section{Author Contributions}

A.L. designed the research; W.Z. contributed analytical tools; A.M.Y. provided LSU data for phylogeny; J.R. created the phylogenetic tree; A.L. and K.S. performed trait measurements; A.L. and M.C.R. wrote the manuscript; all authors contributed to the final version of the manuscript.

\section{Additional Information}

Supplementary information accompanies this paper at https://doi.org/10.1038/s41598-019-50565-7.

Competing Interests: The authors declare no competing interests.

Publisher's note Springer Nature remains neutral with regard to jurisdictional claims in published maps and institutional affiliations.

(c) (1) Open Access This article is licensed under a Creative Commons Attribution 4.0 International License, which permits use, sharing, adaptation, distribution and reproduction in any medium or format, as long as you give appropriate credit to the original author(s) and the source, provide a link to the Creative Commons license, and indicate if changes were made. The images or other third party material in this article are included in the article's Creative Commons license, unless indicated otherwise in a credit line to the material. If material is not included in the article's Creative Commons license and your intended use is not permitted by statutory regulation or exceeds the permitted use, you will need to obtain permission directly from the copyright holder. To view a copy of this license, visit http://creativecommons.org/licenses/by/4.0/.

(C) The Author(s) 2019 